\title{
Geometry, Statistics and Asymptotics of Quantum Pumps
}

\author{
J. E. Avron (a), A. Elgart ${ }^{(a)}$, G.M. Graf ${ }^{(b)}$ and L. Sadun $(a, c)$ \\ (a) Department of Physics, Technion, 32000 Haifa, Israel \\ (b) Theoretische Physik, ETH -Hönggerberg, 8093 Zürich, Switzerland \\ (c) Department of Mathematics, University of Texas, Austin Texas 78712, USA
}

\begin{abstract}
We give a pedestrian derivation of a formula of Büttiker et. al. (BPT) relating the adiabatically pumped current to the $S$ matrix and its (time) derivatives. We relate the charge in BPT to Berry's phase and the corresponding Brouwer pumping formula to curvature. As applications we derive explicit formulas for the joint probability density of pumping and conductance when the $S$ matrix is uniformly distributed; and derive a new formula that describes hard pumping when the $S$ matrix is periodic in the driving parameters.

PACS numbers: 72.10.Bg, 73.23.-b
\end{abstract}

Brouwer [3], and Aleiner et. al. [4], building on results of Büttiker, Pretre and Thomas (BPT) [5], pointed out that adiabatic scattering theory leads to a geometric description of charge transport in mesoscopic quantum pumps. Some of these works, and certainly our own work, was motivated by experimental results of Switkes et. al. [6] on such pumps.

In this article we examine the formula of BPT [0], which relates adiabatic charge transport to the $S$ matrix and its (time) derivatives, in the special case of singlechannel scattering. We show that the formula admits a simple interpretation in terms of three basic processes at the Fermi energy. Two of these are dissipative and nonquantized. The third integrates to zero for any cyclic variation in the system.

Next, we describe the geometric significance of BPT and relate it to Berry's phase [2]. It follows that the pumping formula of Brouwer [3] can be interpreted as curvature and is formally identical to the adiabatic curvature [2]. In spite of the interesting geometry the topological aspects of pumping are trivial. In particular, we prove that all Chern numbers associated to the Brouwer formula are identically zero.

We proceed with two applications. First we give an elementary and explicit derivation of the joint probability density for pumping and conductance. This problem was studied in [3]. Brouwer's results go beyond ours as he also calculates the tails of the distributions and we don't. On the other hand, parts of his results are numerical, and they are certainly not elementary. Finally, we calculate, for the first time, the asymptotics of hard pumping for $S$ matrices that depend periodically on two parameters. If the system traverses a circle of radius $R$ in parameter space, with $R$ large, then the amount of charge transported is order $\sqrt{R}$, multiplied by a quasi-periodic (oscillatory) function of $R$ leading to ergodic behavior.

We shall use units where $e=m=\hbar=1$, so the electron charge is -1 and the quantum of conductance is $e^{2} / h=\frac{1}{2 \pi}$. The mutual Coulombic interaction of the electrons is disregarded.
The BPT formula: Consider a scatterer connected to leads that terminate at electron reservoirs. All the reservoirs are initially at the same chemical potential and at zero temperature. The scatterer is described by its (on-shell) $S$ matrix, which, in the case of $n$ channels is an $n \times n$ matrix parameterized by the energy and other parameters associated with the adiabatic driving of the system (e.g. gate voltages and magnetic fields).

The BPT formula [5] says that the charge $d q_{\ell}$ entering the scatterer from the $\ell$-th lead due to an adiabatic variation of $S$ is

$$
d q_{\ell}=\frac{i}{2 \pi} \operatorname{Tr}\left(Q_{\ell} d S S^{\dagger}\right)
$$

where $Q_{\ell}$ is a projection on the channels in the $\ell$-th lead, and the $S$ matrix is evaluated at the Fermi energy. In the special case of two leads, each lead carrying a single channel,

$$
S=\left(\begin{array}{cc}
r & t^{\prime} \\
t & r^{\prime}
\end{array}\right), \quad Q_{\ell}=\left(\begin{array}{ll}
1 & 0 \\
0 & 0
\end{array}\right)
$$

where $r,\left(r^{\prime}\right)$ and $t,\left(t^{\prime}\right)$ are the reflection and transmission coefficients from the left (right) and $Q_{\ell}$ projects on the left lead. In this case Eq. (1), for the charge entering through the left lead, reduces to

$$
2 \pi d q_{\ell}=i\left(\bar{r} d r+\bar{t}^{\prime} d t^{\prime}\right)
$$

We shall present an elementary derivation of (3).

Derivation: Every unitary $2 \times 2$ matrix can be expressed in the form:

$$
S=e^{i \gamma}\left(\begin{array}{cc}
\cos (\theta) e^{i \alpha} & i \sin (\theta) e^{-i \phi} \\
i \sin (\theta) e^{i \phi} & \cos (\theta) e^{-i \alpha}
\end{array}\right),
$$

where $0 \leq \alpha, \phi<2 \pi, 0 \leq \gamma<\pi$ and $0 \leq \theta \leq \pi / 2$. In terms of these parameters, Eq. (3) reads

$$
2 \pi d q_{\ell}=-\cos ^{2}(\theta) d \alpha+\sin ^{2}(\theta) d \phi-d \gamma .
$$

The basic strategy of our derivation of Eq. (5) is to find processes that vary each of the parameters in turn, and 
keep track of how much current is generated by each process. An underlying assumption is that current depends only on $S\left(k_{F}\right)$ and $\dot{S}\left(k_{F}\right)$, so that processes that give rise to the same change in the $S$ matrix also give rise to the same current. Because we do not prove this assertion, our derivation cannot be considered a complete proof.

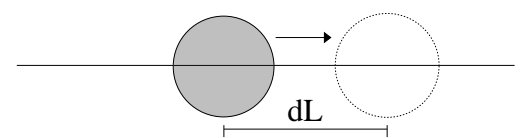

FIG. 1. Moving the scatterer changes $\alpha \rightarrow \alpha+2 k_{F} d L$

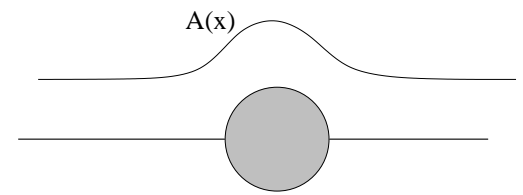

FIG. 2. Applying a vector potential changes $\phi \rightarrow \phi-\int A$

We understand the four parameters as follows. (See figures.) The parameter $\alpha$ is associated with translations: Translating the scatterer a distance $d L=d \alpha / 2 k_{F}$ to the right multiplies $r,\left(r^{\prime}\right)$ by $e^{i d \alpha},\left(e^{-i d \alpha}\right)$, and leaves $t$ and $t^{\prime}$ unchanged. The parameter $\phi$ is associated with a vector potential $A$ near the scatterer. This induces a phase shift $d \phi=-\int A$ across the scatterer, and multiplies $t,\left(t^{\prime}\right)$ by $e^{i d \phi},\left(e^{-i d \phi}\right)$, while leaving $r$ and $r^{\prime}$ unchanged. The parameter $\theta$ determines the conductance of the system: $g=|t|^{2} / 2 \pi=\sin ^{2}(\theta) / 2 \pi$. Finally, $\gamma=(-i / 2) \log \operatorname{det} S$ is related, by Krein's spectral shift [7], to the number of electrons trapped in the scatterer. As a consequence, for any closed path in the space of Hamiltonians $\oint d \gamma=0$.

To determine the effect of changing $\alpha$ we imagine a process that changes $\alpha$ and leaves the other parameters fixed, namely translating the scatterer a distance $d L=d \alpha / 2 k_{F}$ to the right. The scatterer passes through a fraction $|t|^{2}$ of the $k_{F} d L / \pi=d \alpha / 2 \pi$ electrons that occupy the region of size $d L$, and pushes the remaining $|r|^{2} d \alpha / 2 \pi$ electrons forward. Thus

$$
2 \pi d q=-\cos ^{2}(\theta) d \alpha .
$$

This result can be obtained less heuristically, [9], by working in the reference frame of the moving scatterer and integrating the contribution of each wave number from 0 to $k_{F}$. From this one also sees [9] that the rate of dissipation at the reservoirs, $P$, is quadratic in the current $I$, with a coefficient that depends on the dispersion relation. If the dispersion relation is quadratic, then

$$
P=2 \pi I^{2} /\left|r\left(k_{F}\right)\right|^{2} .
$$

To change $\phi$, we vary the vector potential. This induces an EMF of strength $-\int \dot{A}=\dot{\phi}$. The current is simply the voltage times the Landauer conductance $|t|^{2} / 2 \pi$ [8. Integrating over time gives

$$
2 \pi d q=\sin ^{2}(\theta) d \phi
$$

A current $I$ then dissipates energy at the reservoirs at a rate

$$
P=2 \pi I^{2} /\left|t\left(k_{F}\right)\right|^{2}
$$

To understand the effect of changing $\theta$ and $\gamma$, we first suppose our scatterer is right-left symmetric, so $r=r^{\prime}$ and $t=t^{\prime}$. Then changes in $\theta$ and $\gamma$ would draw equal amounts of charge to the scatterer from the left and right leads. The charge that accumulates on the scatterer is given by Krein's spectral shift [7]. The charge coming from the left is half this, namely [9]:

$$
2 \pi d q=\frac{2 \pi i}{4 \pi} d \log \operatorname{det} S=-d \gamma
$$

Since every $S$ matrix can be obtained by translating and adding a vector potential to a right-left symmetric scatterer, the formula (10) applies to all possible $S$-matrices. The formula (10) applies to all possible scatterers, symmetric or not.

Combining (6), (8) and (10), gives BPT, Eq. (5).

The effect of changing $\gamma$ integrates to zero on a closed loop. Changing $\theta$ does not give any transport at all. Thus, only changes in $\alpha$ and $\phi$ contribute to the net transport of a quantum pump. These are dissipative processes, with the rate of energy dissipation $P$ (in the reservoirs) that is bounded from below by $2 \pi I^{2}$. This is contrary to the assertions of [4], who claimed that the charge transport is the sum of a quantized non-dissipative term and a dissipative term that is not quantized.

Geometrical interpretation: $\mathcal{A}=-2 \pi d q$ is the 1form (vector potential) associated with Berry's phase [2]. If we define the unit spinor $|\psi\rangle=\left(\begin{array}{c}r \\ t^{\prime}\end{array}\right)$ then

$$
\mathcal{A}=-i\langle\psi \mid d \psi\rangle
$$

The set of all spinors $|\psi\rangle$ is a 3 -sphere (since $|r|^{2}+\left|t^{\prime}\right|^{2}=$ 1 ), while the set of all ratios $r / t^{\prime}$ is the projective space $\mathbb{C P}^{1}=\mathbb{C}+\{\infty\} \simeq S^{2}$. The natural map between them, namely $|\psi\rangle \rightarrow r / t^{\prime}$, is called the Hopf fibration, and $\mathcal{A}$ is called the "global angular form" of this fibration.

To compute the charge transported by a closed cycle $C$ in parameter space, we can either integrate the 1 -form $\mathcal{A}$ around $C$, or (by Stokes' theorem) integrate the exterior derivative (curl) $\Omega=d \mathcal{A}$ over a disk $D$ whose boundary is $C . \Omega$ is the curvature 2 -form of Brouwer [3]:

$$
\Omega=-i\langle d \psi \mid d \psi\rangle=-i \frac{d \bar{z} \wedge d z}{\left(1+|z|^{2}\right)^{2}},
$$

where $z=r / t^{\prime}$. The expression $-i\langle d \psi \mid d \psi\rangle$ is formally identical to the adiabatic (Berry's) curvature that appears also in the quantum Hall effect [1]. 
In the last expression one sees that the curvature sees only the ratio $z=r / t^{\prime}$, and not $r$ and $t^{\prime}$ separately. The curvature $\Omega$ is the $U(2)$-invariant area form on $\mathbb{C P}^{1}$, and its integral over all of $\mathbb{C P}^{1}$ is $2 \pi . \Omega$ is also the curvature of the Hopf fibration.

In the study of non-dissipative quantum transport, Chern numbers play a role. These are topological invariants that equal the integral of the curvature over closed surfaces in parameter space. In the context of adiabatic scattering, however, all Chern numbers are zero. The vector bundle over parameter space is topologically trivial, and the vector $\left(r, t^{\prime}\right)$ gives a section of this bundle.

These geometrical constructions generalize to systems with $n$ incoming and $m$ outgoing channels, 9. The first $n$ rows of $S$ span an $n$-dimensional complex subspace of $\mathbb{C}^{n+m}$. The space of all such subspaces, called a Grassmannian, has a naturally defined 2-form, called the Kähler form [10]. Up to a constant factor, the Brouwer 2 -form equals the Kähler form. In addition, there is a canonically defined line bundle over this Grassmannian, and $\mathcal{A}$ equals the global angular form for this bundle.

Statistics of weak pumping: Next we consider how a random scatterer transports charge when two parameters are varied gently and cyclically. More precisely, we consider the charge transported by moving along the circle $X_{1}=\epsilon \cos (\tau), X_{2}=\epsilon \sin (\tau)$ in parameter space. If $\epsilon$ is small, then the charge transport is close to $-\frac{\pi \epsilon^{2}}{2 \pi} \Omega\left(\partial_{1}, \partial_{2}\right)$, evaluated at the origin, where $\partial_{j}$ are the tangent vectors associated with the parameters $X_{j}$. The vectors $\partial_{j}$ map to random vectors on $U(2)$, which we assume to be Gaussian with covariance $C$. The problem is then to understand the possible values of the curvature $\Omega$ applied to two random vectors.

To do this, we first need to understand the statistics of 2-forms applied to pairs of random vectors, and to understand the geometry of the group $U(2)$.

Take two random vectors in $\mathbb{R}^{2}$, and see how much area they span. By random vectors we mean independent, identically distributed Gaussian random vectors whose components $X_{j}$ have the covariance $\left\langle X_{i} X_{j}\right\rangle=C \delta_{i j}$. The area $A$ is distributed as a 2 -sided exponential:

$$
d P(A)=\frac{1}{2 C} e^{-|A| / C} d A
$$

This is seen as follows. If the two vectors are $X$ and $Y$, then the area is $X_{1} Y_{2}-X_{2} Y_{1} . X_{1} Y_{2}$ and $-X_{2} Y_{1}$ are independent random variables, and a calculation shows that their characteristic function is $1 / \sqrt{k^{2} C^{2}+1}$. Their sum is a random variable with characteristic function $\left(k^{2} C^{2}+1\right)^{-1}$, and so exponentially distributed.

We parameterize the group $U(2)$ by the angles $(\alpha, \gamma, \phi, \theta)$, as in (4). A standard, bi-invariant metric on $U(2)$ is

$$
\frac{1}{2} \operatorname{Tr}\left(d S^{*} \otimes d S\right)=(d \gamma)^{2}+\cos ^{2} \theta(d \alpha)^{2}+
$$

$$
+\sin ^{2} \theta(d \phi)^{2}+(d \theta)^{2}
$$

In this metric the vectors $\partial_{i}$ are orthogonal but not orthonormal. Unit tangent vectors are

$$
e_{\gamma}=\partial_{\gamma}, e_{\alpha}=\frac{1}{\cos \theta} \partial_{\alpha}, e_{\phi}=\frac{1}{\sin \theta} \partial_{\phi}, e_{\theta}=\partial_{\theta}
$$

The volume form is $\sin (\theta) \cos (\theta) d \alpha \wedge d \gamma \wedge d \phi \wedge d \theta$. The curvature 2-form, from Eq. (12), is

$$
\Omega=-2 \sin (\theta) \cos (\theta) d \theta \wedge(d \alpha+d \phi)
$$

A scattering matrix is time reversal invariant if and only if $t=t^{\prime}$. The space of time-reversal matrices is parameterized exactly as before, only now with $\phi$ identically zero. The volume form for the metric inherited from $U(2)$ is $\cos (\theta) d \alpha \wedge d \gamma \wedge d \theta$, and the curvature form is now $\Omega=-2 \sin (\theta) \cos (\theta) d \theta \wedge d \alpha$.

We are now prepared to compute the statistics of weak pumping, assuming that the $S$ matrix is uniformly distributed and that the tangent vectors to the space of $S$ matrices are Gaussian random variables. This problem was studied by Brouwer [3], in the framework of random matrix theory, by which we mean that Brouwer posits an a-priori measure on the space of Hamiltonians. Random matrix theory is more powerful, in that the distribution of tangent vectors is fixed by the theory. The price one pays is that the analysis is also far from elementary and the results are, in part, only numerical.

For systems without time reversal symmetry, random matrix theory posits that the $S$ matrix is distributed on $U(2)$ with a uniform measure. Since the conductance $g$ is $g \propto|t|^{2}=\sin ^{2} \theta$ we have that $d g \propto \sin \theta \cos \theta d \theta$, proportional to the volume form: The conductance $g$ is therefore uniformly distributed.

A random tangent vector to $U(2)$ is $X=X_{\theta} e_{\theta}+$ $X_{\alpha} e_{\alpha}+X_{\phi} e_{\phi}+X_{\gamma} e_{\gamma}$, where $X_{j}$ are Gaussians with $\left\langle X_{j} X_{k}\right\rangle=C \delta_{j k}$. The curvature associated with two random tangent vectors $X, Y$ is, by Eq. (16)

$$
\Omega(X, Y)=-2\left(X_{\theta} W_{\theta}-Y_{\theta} Z_{\theta}\right)
$$

where $W_{\theta}=\sin \theta Y_{\alpha}+\cos \theta Y_{\phi}$ and $Z_{\theta}=\sin \theta X_{\alpha}+$ $\cos \theta X_{\phi}$. The variables $W_{\theta}$ and $Z_{\theta}$ are independent, each with variance $C$. From Eq. (13), the distribution of the curvature is exponential and independent of $|t|$. The joint distribution of curvature, $\omega$, and conductance, $g=\frac{1}{2 \pi}|t|^{2}$ is given by the probability density

$$
\frac{\pi}{2 C} e^{-|\omega| / 2 C} d \omega d g
$$

with $\omega$ ranging from $-\infty$ to $\infty$ and $g$ from 0 to $\frac{1}{2 \pi}$.

For systems with time reversal symmetry, the $S$ matrix is uniformly distributed on the $t=t^{\prime}$ submanifold, with the metric inherited from $U(2)$. The tangent vectors are now Gaussian random variables of the form $X=X_{\theta} e_{\theta}+X_{\alpha} e_{\alpha}+X_{\gamma} e_{\gamma}$, and the curvature is now 


$$
\Omega(X, Y)=-2 \sin \theta\left(X_{\theta} Y_{\alpha}-Y_{\theta} X_{\alpha}\right) .
$$

Since the curvature depends on $\theta$ the curvature and the conductance are correlated. The volume form indicates that $\sqrt{g}$, and not $g$, is uniformly distributed. This favors insulators. The joint distribution for curvature and conductance is

$$
\frac{1}{4 \sqrt{g} C} e^{-|\omega| / 2 C \sqrt{2 \pi g}} d \omega d(\sqrt{g}) .
$$

This formula says that, statistically, good pumps tend to be good conductors; $\omega / \sqrt{g}$, rather than $\omega$ itself, is independent of $g$.

We have assumed, so far, that the variance $C$ is a constant. There is no reason for this and it is natural to let $C$ itself be a random variable. Given a probability distribution for the covariance, $d \mu(C)$, one integrates the formulas (18) and (20) over $C$. One sees, by inspection, that in the absence (presence) of time reversal symmetry, $\omega(\omega / \sqrt{g})$ is independent of $g$. Furthermore, the distribution of $\omega$ after integrating over $g$ is smooth away from $\omega=0$, but has a discontinuity in derivative (log divergence) at $\omega=0$. In these qualitative features, our results agree with Brouwer's. However, the tails of the distribution for large pumping may depend on the tail of $d \mu(C)$; While (18) and (20) have exponentially small tails, power law tails $d \mu(C)$ will lead to power law in the tails in $\omega$. Since we do not determine $d \mu(C)$ we can not determine the tails. Using random matrix theory Brouwer determined the power decay in $\omega[3]$.

Hard Pumping: Finally, we consider what happens for hard pumping. Here one can no longer evaluate the curvature at a point and multiply by the area. One needs to honestly integrate the curvature. Hard pumping was addressed by [4] who studied it in the context of random matrix theory and showed, using rather involved diagrammatic techniques, that pumping scales like the root of the perimeter. Here we shall describe a complementary, elementary result that holds provided the $S$ matrix is a periodic function of the parameters. This is the case, for example, when the pumping is driven by two Aharonov-Bohm fluxes.

With $S(x, y)$ periodic in the driving parameters $x$ and $y$, so is the curvature $\Omega(x, y)=\sum \hat{\Omega}_{m n} e^{i(m x+n y)}$. Since the global angular form is also periodic, $\hat{\Omega}_{00}=0$.

The integral $\int_{|x|<R} \Omega$ of the curvature on a large disc of radius $R$ is, to leading order,

$$
\sqrt{8 \pi R} \sum \frac{\hat{\Omega}_{n m}}{N(n, m)^{3 / 2}} \sin \left(N(n, m) R-\frac{\pi}{4}\right),
$$

where $N(n, m)=\sqrt{n^{2}+m^{2}}$. The charge transported in a cycle is proportional to the square root of the perime- ter (or the fourth root of the area) times a quasiperiodic function of $R$. This follows from the evaluation of the elementary integral $\int_{|x|<R} d x d y e^{i(n x+m y)}$, which equals a Bessel function whose large- $R$ asymptotic behavior is $\sqrt{\frac{8 \pi R}{N^{3}}} \sin \left(N R-\frac{\pi}{4}\right)$. From Eq. (21) one can determine the probability distribution for charge transport, (viewed as a random variable with uniform distribution on the radius $R$ ). Eq. (21) turns out to be closely related to a celebrated problem in ergodic number theory: The Gauss circle problem [11].

This result does not directly apply to the pump studied by Switkes [6], because the parameters they vary do not have built in periodicity. Nevertheless, it illustrates two features of pumps that have been observed experimentally. The first is that hard driving transports a lot of charge, with scaling that is sublinear, as in a random process, and the second that the directionality of hard pumping is essentially unpredictable.

We thank A. Kamenev for extremely valuable insights and A. Auerbach, P. Brouwer and C. Marcus for useful correspondences, and U. Sivan for a careful reading of the manuscript, and valuable suggestions. This research was supported in part by the Israel Science Foundation, the Fund for Promotion of Research at the Technion, the DFG, the National Science Foundation and the Texas Advanced Research Program.

[1] D. J. Thouless, Topological quantum numbers in nonrelativistic physics, World Scientific, Singapore, 1998.

[2] M.V. Berry, Proc. Roy. Soc. London A 39245 (1984).

[3] P.W. Brouwer, Phys. Rev. B 58, 10135 (1998).

[4] T.A. Shutenko, I.L. Aleiner, B.L. Altshuler, cond-mat/9911019.

[5] M. Büttiker, H. Thomas, A. Prêtre, Z. Phys. B 94, 133 (1994).

[6] M. Switkes, C.M. Marcus, K. Campman, and A.G. Gossard, Science 283, 1907 (1999).

[7] D.R. Yafaev, Mathematical Scattering Theory, AMS (1992).

[8] Y. Imry, Introduction to mesoscopic physics, Oxford University Press (1997).

[9] J. Avron, A. Elgart, G.M. Graf and L. Sadun, in preparation.

[10] S. Kobayashi and K. Nomizu, Foundations fo Differential Geometry, Wiley(1963).

[11] P.M. Bleher, Z. Cheng, F.J. Dyson and J.L. Lebowitz, Comm. Math. Phys. 154, 433 (1993) 